\title{
On the existence of angular limits of $n$-dimensional quasiconformal mappings
}

\author{
Matti Vuorinen
}

\section{Introduction}

Let $G$ and $G^{\prime}$ be domains in the euclidean space $\mathbf{R}^{n}, n \geqq 2$, let $f: G \rightarrow G^{\prime}$ be a homeomorphism, and for $x \in G$ and $r \in(0, d(x, \partial G))$ let $L(x, r)=\max \{|f(x)-f(y)|$ : $|x-y|=r\}$ and let $l(x, r)=\min \{|f(x)-f(y)|:|x-y|=r\}$. The mapping $f$ is quasiconformal if there exists a finite constant $K \geqq 1$ such that

$$
\limsup _{r \rightarrow 0+} \frac{L(x, r)}{l(x, r)} \leqq K
$$

for every $x \in G$. The smallest possible $K \geqq 1$ is called the dilatation of the mapping $f$. It measures the distortion of small spheres under the mapping and hence indicates the amount of deviation of the mapping from a conformal mapping, whose dilatation is one. For basic properties of quasi conformal mappings we refer the reader to the book of Väisälä [14].

An important boundary property of quasiconformal mappings is given in the following theorem of $\mathrm{F}$. W. Gehring [3, p. 21]: If a quasiconformal mapping of the unit ball $B^{n}$ has an asymptotic value $\alpha$ at a point $b \in \partial B^{n}$, then the mapping has the angular limit $\alpha$ at $b$. This result generalizes Lindelöf's classical theorem about angular limits of conformal mappings of the unit disc. The purpose of this paper is to show that actually much stronger Lindelöf-type theorems than Gehring's theorem hold for quasiconformal mappings.

The fundamental problem to be studied is the following. Let $f: B^{n} \rightarrow G^{\prime}$ be a quasiconformal mapping, let $b \in \partial B^{n}$, and let $E$ be a subset of $B^{n}$ with $b \in \bar{E}$. Suppose that $f(x)$ tends to a limit $\alpha$ as $x$ approaches $b$ through the set $E$. How thick must the set $E$ be at $b$ in order that $f$ have the angular limit $\alpha$ at $b$ ? By Gehring's theorem this is the case if $E$ is a curve converging to $b$. We shall give a substantially weaker sufficient density condition on $E$ which only requires that the lower (conformal) 
capacity density of $E$ be positive at $b$. We shall prove the existence of angular limits by means of a normal family argument. An important subclass of two-dimensional quasiconformal mappings is the class of conformal mappings, i.e. the class of univalent analytic functions. It is likely that our main results are new in this particular case, too. In any case, the main theorems, Theorems 4.4 and 5.5, seem to be among the best results also in the case of univalent analytic functions.

Some crucial tools, the capacity and radial densities, are introduced and studied in Sections 1 and 2. Section 3 contains a normal family criterion for the existence of an angular limit. These three sections constitute the introductory part of the paper, on which the subsequent theory is based.

In Section 4 we prove the result mentioned above, i.e. that $\alpha$ is an angular limit of a quasiconformal mapping $f$ of $B^{n}$ at $b \in \partial B^{n}$ if $f(x)$ tends to $\alpha$ as $x$ approaches $b$ through a set $E$ having positive lower capacity density at $b$. In Section 6 we shall show that the condition on $E$ is, in a sense, best possible. One consequence of that result extends a Lindelöf-type theorem of T. Hall [5, Thm. II] concerning bounded analytic functions.

The main results of the paper are given in Section 5. There we extend a very general Lindelöf-type theorem of J. L. Doob [2, Thm. 4] to the case of quasiconformal mappings. Finally, in Section 6 we consider the case where a quasiconformal mapping of $B^{n}$ tends to a constant limit function $\alpha$ through a sequence of points in $B^{n}$ converging to a point $b \in \partial B^{n}$. With the aid of the hyperbolic metric we give a density condition on the sequence guaranteeing that $\alpha$ is the angular limit at $b$. As a corollary we obtain a quasiconformal analogue to a theorem of Bagemihl and Seidel [1, Thm. 1].

A sense-preserving mapping $f: B^{n} \rightarrow \mathbf{R}^{n}$ is quasiconformal if and only if it is injective and quasiregular in the sense of Martio, Rickman, and Väisälä [7] and [8]. Hence it is of interest to study whether the results of this paper can be extended to the case of $n$-dimensional quasiregular mappings. With minor modifications in the proofs one can show that the main theorems, Theorems 4.4 and 5.5, hold for such a subclass of quasiregular mappings that each mapping in the subclass is closed in the sense of [15, Ch. II]. Such results were announced in [16]. The assumption that a quasiregular mapping be closed is very restrictive, however. Thus an important recent example of S. Rickman shows that not even Lemma 4.1, and a fortiori Theorems 4.4 and 5.5, need hold for bounded quasiregular mappings of $B^{3}$. For some positive results concerning angular limits of quasiregular mappings the reader is referred to [17], where also references to Rickman's work can be found.

Notation. We denote the $n$-dimensional euclidean space, $n \geqq 2$, by $\mathbf{R}^{n}$, and its one-point compactification by $\overline{\mathbf{R}}^{n}=\mathbf{R}^{n} \cup\{\infty\}$. The space $\mathbf{R}^{n}$ is equipped with the inner product $(x \mid y)=\sum_{i=1}^{n} x_{i} y_{i}$ and with the metric given by the norm $|x|=(x \mid x)^{1 / 2}$. 
All topological operations are performed with respect to $\overline{\mathbf{R}}^{n}$ if not otherwise indicated. Balls and spheres centered at $x \in \mathbf{R}^{n}$ and with radius $r>0$ are denoted by $B^{n}(x, r)=\left\{z \in \mathbf{R}^{n}:|z-x|<r\right\}$ and $S^{n-1}(x, r)=\left\{z \in \mathbf{R}^{n}:|z-x|=r\right\}$. We employ the abbreviations:

$$
\begin{gathered}
B^{n}(r)=B^{n}(0, r), \quad B^{n}=B^{n}(1), \\
S^{n-1}(r)=S^{n-1}(0, r), \quad S^{n-1}=S^{n-1}(1) .
\end{gathered}
$$

The standard coordirate unit vectors are $e_{i}, i=1, \ldots, n$.

\section{Density in the n-capacity}

We shall introduce in this section the lower and upper capacity densities of a set and study their properties. The results will usually be formulated for lower capacity densities only, since they will play an important role in Sections 4-6. Their obvious analogues hold for upper capacity densities as well.

First we give some preliminary definitions and remarks.

Definition 1.1. The modulus of a path family. A path is a continuous nonconstant mapping $\gamma: \Delta \rightarrow A, A \subset \overline{\mathbf{R}}^{n}$, where $\Delta$ is an interval on the real axis. The point set $\gamma \Delta$ is denoted by $|\gamma|$. The modulus of a path family $\Gamma$ is defined by

$$
M(\Gamma)=\inf _{\varrho \in F(\Gamma)} \int_{\mathbf{R}^{n}} \varrho^{n} d m_{n} .
$$

Here $m_{n}$ is $n$-dimensional Lebesgue measure and $F(\Gamma)$ is the family of all non-negative Borel functions $\varrho: \mathbf{R}^{n} \rightarrow \mathbf{R}^{1} \cup\{\infty\}$ such that

$$
\int_{\gamma} \varrho d s \geqq 1
$$

for every locally rectifiable path $\gamma$ in $\Gamma$. An important property of the modulus is that it is an outer measure on the collection of all path families of $\mathbf{R}^{n}$. For this and other properties of the modulus we refer the reader to the book of Väisälä [14, Ch. 1].

Definition 1.2. Quasiconformal mappings. Instead of using directly the metric definition of quasiconformality given in the Introduction, we shall employ an alternative equivalent definition, involving moduli of path families. Accordingly, a homeomorphism $f: G \rightarrow G^{\prime}$ of a domain $G \subset \overline{\mathbf{R}}^{n}$ onto a domain $G^{\prime} \subset \overline{\mathbf{R}}^{n}$ is called quasiconformal if there is a finite constant $K \geqq 1$ such that for every path family $\Gamma$ whose elements lie in $G$

$$
\frac{M(\Gamma)}{K} \leqq M(f \Gamma) \leqq K M(\Gamma)
$$


Here $f \Gamma=\{f \circ \gamma: \gamma \in \Gamma\}$. The smallest possible $K \geqq 1$ for which (1.3) holds is denoted by $K(f)$.

Definition 1.4. Condensers and their capacity. A condenser is a pair $(A, C)$ where $A$ is open in $\mathbf{R}^{n}$ and $C$ is a compact non-empty subset of $A$. Given sets $E, F$, and $G$ in $\overline{\mathbf{R}}^{n}$ we let $\Delta(E, F ; G)$ denote the family of all paths $\gamma:[0,1] \rightarrow \overline{\mathbf{R}}^{n}$ with $\gamma(0) \in E, \gamma(1) \in F$, and $\gamma(t) \in G$ for $t \in(0,1)$. The capacity, or (conformal) n-capacity, of a condenser $(A, C)$ is defined by

$$
\operatorname{cap}(A, C)=M\left(\Delta\left(C, \partial A ; \overline{\mathbf{R}}^{n}\right)\right)=M(\Delta(C, \partial A ; A)) .
$$

A compact set $F$ in $\overline{\mathbf{R}}^{n}, F \neq \overline{\mathbf{R}}^{n}$, is said to be of capacity zero if the modulus of the family of all paths $\gamma$ in $\overline{\mathbf{R}}^{n}$ with $|\bar{\gamma}| \cap F \neq \emptyset$, is zero. An arbitrary set $F \subset \mathbf{R}^{n}$ is said. to be of capacity zero if every compact subset of $F$ is of capacity zero. Otherwise $F$ is of positive capacity. We write cap $F=0$ or cap $F>0$, respectively.

Definition 1.6. Capacity densities. Given a subset $E$ of $\mathbf{R}^{n}$, a point $x$ in $\mathbf{R}^{n}$, and numbers $t>r>0$, we introduce the following abbreviations:

$$
\begin{gathered}
M_{t}(E, r, x)=M\left(\Delta\left(S^{n-1}(x, t), \bar{B}^{n}(x, r) \cap E ; \overline{\mathbf{R}}^{n}\right)\right), \\
M(E, r, x)=M_{2 r}(E, r, x) .
\end{gathered}
$$

For $t>s>r>0$ the following estimates hold

$$
M_{t}(E, r, x) \leqq M_{s}(E, r, x) \leqq\left[\frac{\log \frac{t}{r}}{\log \frac{s}{r}}\right]^{n-1} \cdot M_{t}(E, r, x) .
$$

The first inequality is obvious (cf. $[14,6.4]$ ) and the second one is due to Martio and Sarvas $[9,2.7]$. The lower and upper capacity densities of $E$ at $x$ are defined, respectively, by

$$
\begin{aligned}
& \text { cap dens }(E, x)=\liminf _{r \rightarrow 0} M(E, r, x), \\
& \text { cap dens }(E, x)=\limsup _{r \rightarrow 0} M(E, r, x) .
\end{aligned}
$$

Both of these quantities are bounded and non-negative. In fact, cap $\overline{\operatorname{den} s}(E, x) \leqq$ $\omega_{n-1}(\log 2)^{1-n}$ holds for every $x \in \mathbf{R}^{n}$. Here $\omega_{n-1}$ is the $(n-1)$-dimensional surface area of $S^{n-1}$. The upper bound follows from the useful estimate

$$
M(\Gamma) \leqq \omega_{n-1}\left(\log \frac{b}{a}\right)^{1-n},
$$

which holds if $0<a<b$ and $\Gamma$ is a path family in $\overline{\mathbf{R}}^{n}$ such that, for some $u \in \mathbf{R}^{n}$, $|\gamma|$ intersects both boundary components of $B^{n}(u, b) \backslash \bar{B}^{n}(u, a)$ for all $\gamma \in \Gamma$ (cf. $[14,7.5]$ ). 
Remarks 1.9.

(1) The $n$-capacity of a condenser $(A, C)$ is often defined as

$$
\operatorname{cap}(A, C)=\inf _{u} \int_{A}|\nabla u|^{n} d m_{n}
$$

where $u$ runs through all $C^{\infty}$-functions with compact support in $A$ such that $u(x) \geqq 1$ for $x \in C$. It follows from the results of Ziemer in [19] that this alternative definition is equivalent to (1.5).

(2) Martio and Sarvas have studied in [9] the condition cap $\overline{\operatorname{dens}}(E, x)=0$ and other related conditions for compact sets $E$. For our purposes the condition cap dens $(E, x)>0$ will be of interest. We refer the reader to Examples 2.5, where the properties of capacity densities will be illustrated.

In the sequel we shall need lower bounds for cap dens $(E, 0)$ when $E \subset \mathbf{R}^{n}$. The following lemma, which is based on the spherical cap-inequality of Gehring (cf. Väisälä $[14,10.2,10.9,10.12]$ ), is then often needed. Throughout the entire paper we denote by $c_{n}$ the positive constant in [14, 10.9], which depends only on the dimension $n$. We denote by $d(A)$ the euclidean diameter of a set $A \subset \mathbf{R}^{n}$.

Lemma 1.10. Let $E \subset \mathbf{R}^{n}$ and $t>r>0$. Suppose that there is a connected set $E_{r} \subset \bar{B}^{n}(r) \cap E$. Then

$$
M_{t}(E, r, 0) \geqq c_{n} \log \frac{2 t+d\left(E_{r}\right)}{2 t-d\left(E_{r}\right)} .
$$

If $\bar{E}_{r} \cap S^{n-1}(r) \neq \emptyset$ and $\bar{E}_{r} \cap S^{n-1}(s) \neq \emptyset$ for some $s \in(0, r)$, then

$$
M_{t}(E, r, 0) \geqq c_{n} \log \frac{t-s}{t-r} .
$$

Proof. To prove (i), fix $u, v \in \bar{E}_{r}$ with $|u-v|=d\left(E_{r}\right)=d$ and choose a line $L$ through $u$ and $v$. Let $w \in S^{n-1}(t) \cap L$ be such that $p=|v-w| \leqq|u-w|$ and let $y$ be the other point in $S^{n-1}(t) \cap L$ and write $q=|u-y|$. If we apply the spherical capinequality [14, 10.12] in the ring domains $R(w, p+d, p)$ and $R(y, q+d, q)$ we get

$$
\begin{gathered}
M_{t}(E, r, 0) \geqq c_{n} \max \left\{\log \frac{p+d}{p}, \log \frac{q+d}{q}\right\} \\
\geqq c_{n} \max \left\{\log \frac{p+d}{p}, \log \frac{2 t-p}{2 t-p-d}\right\} \geqq c_{n} \log \frac{2 t+d}{2 t-d} .
\end{gathered}
$$

In the middle inequality we have used the estimate $q \leqq 2 t-p-d$.

To prove the second lower bound fix $u \in \bar{E}_{r} \cap S^{n-1}(s)$ and $v \in \bar{E}_{r} \cap S^{n-1}(r)$ and choose a line $L$ through $u$ and $v$. Let $w \in L \cap S^{n-1}(t)$ be such that $|v-w| \leqq|u-w|$. Let $p$ and $q$ denote the lengths of the projections of $u-v$ and $v-w$ on the line 
through 0 and $v$. By the spherical cap-inequality $[14,10.12]$ we obtain

$$
M_{t}(E, r, 0) \geqq c_{n} \log \frac{|u-v|+|v-w|}{|v-w|}=c_{n} \log \left(\frac{p}{q}+1\right) \geqq c_{n} \log \frac{t-s}{t-r},
$$

where we have used the estimate $p / q \geqq(r-s) /(t-r)$.

The next lemma is well known. Actually, it was a part of the proof of Lemma 3.11 in the paper [8] of Martio, Rickman, and Väisälä, but it was not formulated explicitly there. In its present form it was given by Näkki [11,3.3], and following his terminology we shall call it the comparison principle for the modulus.

Lemma 1.11. Let $F_{1}, F_{2}$, and $F_{3}$ be three sets in $\overline{\mathbf{R}}^{n}$ and let $\Gamma_{i j}=\Delta\left(F_{i}, F_{j} ; \overline{\mathbf{R}}^{n}\right)$, $1 \leqq i, j \leqq 3$. If there exist $x \in \mathbf{R}^{n}$ and $0<a<b$ such that $F_{1}, F_{2} \subset \bar{B}^{n}(x, a)$ and $F_{3} \subset$ $\overline{\mathbf{R}}^{n} \backslash B^{n}(x, b)$, then

$$
M\left(\Gamma_{12}\right) \geqq 3^{-n} \min \left\{M\left(\Gamma_{13}\right), M\left(\Gamma_{23}\right), c_{n} \log \frac{b}{a}\right\}
$$

Using Lemmas 1.10 and 1.11 we prove the following result. A continutum is a compact connected subset of $\overline{\mathbf{R}}^{n}$ containing at least two distinct points.

Lemma 1.12. Let $E \subset \overline{\mathbf{R}}^{n}$ be a set with $0 \in \bar{E}$ and let $h_{r}: \mathbf{R}^{n} \rightarrow \mathbf{R}^{n}$ be the dilation $h_{r}(x)=x / r$ for $x \in \mathbf{R}^{n}$ and $r \in(0,1)$. Let $F \subset \bar{B}^{n}(s), s>0$, be a continuum and let $\Gamma_{\mathbf{r}}=\Delta\left(F, \bar{B}^{n}(s) \cap h_{\mathbf{r}} E ; \mathbf{R}^{n}\right)$ for $r \in(0,1)$. Then there is a constant $\mu>0$ depending only on $n$ and $d(F) / s$ such that

$$
\liminf _{r \rightarrow 0} M\left(\Gamma_{r}\right) \geqq \mu \text { cap dens }(E, 0) \text {. }
$$

Proof. Choose $r_{0}>0$ so that $M\left(h_{r} E, s, 0\right) \geqq \delta / 2$ for $r \in\left(0, r_{0}\right]$ where $\delta=$ cap dens $(E, 0)$. This is possible by the definition of cap dens and by [14, 8.1]. Fix $r \in\left(0, r_{0}\right]$. We shall now apply the comparison principle for the modulus, Lemma 1.11 , with $F_{1}=\bar{B}^{n}(s) \cap h_{r} E, F_{2}=F$, and $F_{3}=S^{n-1}(2 s)$. Using the notation of Lemma 1.11 we get by Lemma 1.10

$$
M\left(\Gamma_{23}\right) \geqq c_{n} \log \frac{4 s+d}{4 s-d},
$$

where $d=d(F)$. Since $M\left(\Gamma_{13}\right)=M\left(h_{r} E, s, 0\right) \geqq \delta / 2$, Lemma 1.11 yields in view of $(1.8)$

$$
\begin{aligned}
M\left(\Gamma_{r}\right) & =M\left(\Gamma_{12}\right) \geqq 3^{-n} \min \left\{M\left(\Gamma_{13}\right), M\left(\Gamma_{23}\right), c_{n} \log 2\right\} \\
& \geqq 3^{-n} \min \left\{\frac{\delta}{2}, m\right\} \geqq 3^{-n} \frac{\delta}{2} \min \left\{1, \frac{m}{D}\right\}
\end{aligned}
$$

where $m=\min \left\{c_{n} \log ((4 s+d) /(4 s-d)), c_{n} \log 2\right\}>0$ and $D=\omega_{n-1}(\log 2)^{1-n}$. Since $r \in\left(0, r_{0}\right]$ was arbitrary, we may choose $\mu=\left(2 \cdot 3^{n}\right)^{-1} \min \{1, m / D\}$. 
Definition 1.13. Conformal invariance of capacity densities. A $C^{1}$-homeomorphism $f: D \rightarrow D^{\prime}$, where $D$ and $D^{\prime}$ are domains in $\overline{\mathbf{R}}^{n}$, is conformal if for all $x \in D \backslash\left\{\infty, f^{-1}(\infty)\right\}$ and for all $h \in \mathbf{R}^{n}$

$$
\left|f^{\prime}(x) h\right|=\left|f^{\prime}(x)\right||h|
$$

and the Jacobian determinant $J_{f}(x)$ is non-zero for all $x \in D \backslash\left\{\infty, f^{-1}(\infty)\right\}$. Here $\left|f^{\prime}(x)\right|=\max \left\{\left|f^{\prime}(x) e\right|:|e|=1\right\}$.

An important property of the modulus of a path family is its invariance under conformal mappings. In the next lemma we shall see that the capacity density has a similar property as well.

Lemma 1.14. Let $f: D \rightarrow D^{\prime}$ be conformal, let $E$ be a subset of $D$, let $0 \in D$, and let $f(0) \neq \infty$. Then

$$
\text { cap dens }(E, 0)=\text { cap dens }(f E, f(0)) \text {. }
$$

Proof. The proof is a rather straightforward calculation, where one makes use of $[14,8.1],(1.7)$, and the differentiability of $f$ and $f^{-1}$. The details are left to the reader.

Remark 1.15. One can improve some lower bounds in Sections 1 and 2 by making use of estimates involving the capacity of the Grötzsch ring in place of the spherical cap-inequality (cf. [14, Section 11]).

\section{The radial density}

In the present section we shall derive certain lower bounds for the lower capacity density of a set. Clearly we may expect significant lower bounds only at points where the set is thick in some suitable sense. A relevant condition of this kind, from the point of view of applications in Sections 4-6, can be given in terms of the lower radial density, which will be introduced in this section. The estimates obtained in this way depend crucially on Gehring's spherical cap-inequality (cf. Väisälä $[14,10.2,10.9])$, and on the fact that the capacity of a condenser decreases under symmetrization (cf. Sarvas [13]).

Definition 2.1. Radial densities. Let $m$ be Lebesgue measure on the real axis. Given a set $E$ in $\mathbf{R}^{n}$ and $x \in \mathbf{R}^{n}$ let $A_{x}=\left\{r>0: S^{n-1}(x, r) \cap E \neq \emptyset\right\}$. If $A_{x}$ is measurable we define the lower and upper radial density of $E$ at $x$ respectively by

$$
\begin{aligned}
& \operatorname{rad} \underline{\text { dens }}(E, x)=\liminf _{r \rightarrow 0} m\left(A_{x} \cap(0, r)\right) r^{-1}, \\
& \operatorname{rad} \overline{\operatorname{dens}}(E, x)=\limsup _{r \rightarrow 0} m\left(A_{x} \cap(0, r)\right) r^{-1} .
\end{aligned}
$$


It is immediately seen that if $E$ is open or compact then these densities are defined at every point of $E$. Radial densities give information only about the radial distribution of the set $E$ in small neighborhoods of $x$. Therefore it is not possible to obtain upper bounds for capacity densities in terms of radial ones, as Example 2.5 (1) shows. The next lemma provides us with a lower bound of this type.

Lemma 2.2. Let $E$ be an open or compact subset of $\mathbf{R}^{n}$ with $0 \in \bar{E}$ and let $A=\left\{r>0: S^{n-1}(r) \cap E \neq \emptyset\right\}$. Then for $r>0$

$$
M(E, r, 0) \geqq c_{n} \log \frac{2 r}{2 r-m(A \cap(0, r))}
$$

where $c_{n}>0$ is as in $[14,10.9]$. In particular, if rad dens $(E, 0)=\delta$, then

$$
\text { cap dens }(E, 0) \geqq c_{n} \log \frac{2}{2-\delta} \text {. }
$$

Proof. Fix $r>0$. If $m(A \cap(0, r))=0$ there is nothing to prove. Assume $m(A \cap(0, r))>0$ and let $s \in(0, m(A \cap(0, r)))$. Choose a compact set $F \subset\left\{t e_{1}: 0 \leqq t \leqq r\right.$, $t \in A\}$ with $m(F) \geqq s$. By symmetry it follows that $M(E, r, 0) \geqq M(F, r, 0)$; see Sarvas [13] and Ziemer [19]. This combined with the cap-inequality [14, 10.9] yields

$$
M(E, r, 0) \geqq c_{n} \log \frac{2 r}{2 r-s}
$$

Since $s \in(0, m(A \cap(0, r)))$ was arbitrary, the desired estimate follows.

Definition 2.3. Lebesgue densities. In a $k$-dimensional, $1 \leqq k \leqq n$, affine subspace of $\mathbf{R}^{n}$ we employ $k$-dimensional Lebesgue measure $m_{k}$. If $E$ is a measurable set in a $k$-dimensional affine subspace of $\mathbf{R}^{n}$ and $x \in \mathbf{R}^{n}$, then we define the lower and upper Lebesgue density of $E$ at $x$ respectively by

$$
\begin{aligned}
& \Theta_{*}^{k}(E, x)=\liminf _{r \rightarrow 0} m_{k}\left(E \cap \bar{B}^{n}(x, r)\right) r^{-k} \alpha(k)^{-1,} \\
& \Theta^{k_{*}}(E, x)=\limsup _{r \rightarrow 0} m_{k}\left(E \cap \bar{B}^{n}(x, r)\right) r^{-k} \alpha(k)^{-1},
\end{aligned}
$$

where $\alpha(k)=m_{k}\left(\bar{B}^{k}\right)$. If these densities are equal, then this common value is called the Lebesgue density of $E$ at $x$, and it is denoted by $\Theta^{k}(E, x)$. The following lemma gives a relation between the Lebesgue and radial densities.

Lemma 2.4. Let $E$ be an open or compact subset of $\mathbf{R}^{n}$ with $0 \in \bar{E}$, let $V$ be the $k$-dimensional affine subspace through $0,1 \leqq k \leqq n$, and let $p: \mathbf{R}^{n} \rightarrow V$ be an orthogonal projection. If $\Theta_{*}^{k}(p E, 0)=\delta$ then

$$
\operatorname{rad} \underline{\operatorname{dens}}(p E, 0) \geqq 1-\sqrt[k]{1-\delta}
$$


and

$$
\text { cap dens }(E, 0) \geqq c_{n} \log \frac{2}{1+\sqrt[k]{1-\delta}}
$$

where $c_{n}>0$ is as in $[14,10.9]$.

Proof. The proof is a rather straightforward argument, where one makes use of Fubini's theorem, symmetrization, and Lemma 2.2. The details are left to the reader.

Next we give some examples illustrating the interrelations between radial and capacity densities. The third example concerns the condition cap dens $(E, 0)>0$ and shows that there are very thin sets satisfying this condition.

Examples 2.5 .

(1) Let $S_{k}=S^{n-1}\left(2^{-k}\right) \cap\left\{x: x_{n} \geqq 0\right\}, k=1,2, \ldots$ and let $E=\{0\} \cup\left(\cup S_{k}\right)$. By Lemma 1.10 we get cap $\overline{\operatorname{dens}}(E, 0) \geqq c_{n} \log 3$ and cap dens $(E, 0) \geqq c_{n} \log (5 / 3)$ while $\operatorname{rad} \overline{\operatorname{dens}}(E, 0)=0$.

(2) Let $\left(x_{k}\right)$ be a sequence in $\mathbf{R}^{n} \backslash\{0\}$ tending to 0 and let $\left(r_{k}\right)$ be a sequence of positive real numbers such that $\lim \sup r_{k} /\left|x_{k}\right|>0$. Write $E=\{0\} \cup\left(\cup \bar{B}^{n}\left(x_{k}, r_{k}\right)\right)$. Then by the definition $\operatorname{rad} \overline{\operatorname{dens}}(E, 0)>0$ and hence cap $\overline{\operatorname{dens}}(E, 0)>0$ by Lemma 2.2.

(3) With the aid of a theorem of $\mathrm{H}$. Wallin [18] we shall now construct a compact set $E$ with cap dens $(E, 0)>0$ but such that $E$ is of zero Hausdorff dimension. By Wallin's Theorem 4.4 there is a compact Cantor-type set $E_{1}$ of positive capacity and of zero Hausdorff dimension. We may choose $E_{1} \subset B^{n}(2) \backslash \bar{B}^{n}(1)$. Let $h$ : $\mathbf{R}^{n} \rightarrow \mathbf{R}^{n}$ be the mapping $h(x)=x / 4$ and let $E_{k+1}=h E_{k}, k=1,2, \ldots$. The set $E=\{0\} \cup\left(\cup E_{k}\right)$ is compact and of zero Hausdorff dimension. Since cap $E_{1}>0$ also $M\left(\Delta\left(S^{n-1}(8), E_{1} ; \overline{\mathbf{R}}^{n}\right)\right)=\delta>0$, see e.g. Ziemer [19]. This implies cap dens $(E, 0) \geqq \delta$.

\section{Normal functions and angular limits}

The concept of a normal meromorphic function was introduced by Lehto and Virtanen in [6], where they established several important properties of these functions. We shall extend here the definition of normality to the case of mappings defined on a ball or a half space of $n$-dimensional euclidean space. Following the ideas of [6] we shall show that a normal function has an angular limit if and only if certain normal families converge towards a constant mapping. This criterion will play a significant role in Sections 4-6. 
Definition 3.1. Asymptotic values and angular limits. We let the notation $f: G \rightarrow \overline{\mathbf{R}}^{\mathfrak{n}}$ include the assumption that $f$ is continuous and $G$ is a domain in $\overline{\mathbf{R}}^{\mathbf{n}}$. The mapping $f$ has an asymptotic value $\alpha$ at $b \in G$ if there exists a path $\gamma:[0,1) \rightarrow G$ such that $\gamma(t) \rightarrow b$ and $f(\gamma(t)) \rightarrow \alpha$ as $t \rightarrow 1$. For $b \in \partial B^{n}$ and $\varphi \in(0, \pi / 2)$ we let $K(b, \varphi)$ denote the cone $\left\{z \in \mathbf{R}^{n}:(b \mid b-z)>|b-z| \cos \varphi\right\}$. A mapping $f: B^{n} \rightarrow \overline{\mathbf{R}}^{n}$ has an angular limit $\alpha$ at $b \in \partial B^{n}$ if for each $\varphi \in(0, \pi / 2) f(x)$ tends to $\alpha$ as $x$ approaches $b$ through the cone $K(b, \varphi)$. In an obvious way this definition is extended for mappings defined on a half space $\mathbf{R}_{+}^{n}=\left\{x: x_{n}>0\right\}$.

Remark 3.2. Let $f: B^{n} \rightarrow \overline{\mathbf{R}}^{n}$ be a mapping, let $b \in \partial B^{n}$, and let $E \subset B^{n}$ be a set with $b \in \bar{E}$. In Section 4 we shall consider the situation where $f(x)$ tends to a limit $\alpha$ as $x$ approaches $b$ through the set $E$. The following observation is then sometimes useful: There is an open set $F$ containing $\bar{E} \cap B^{n}$ such that $f(x)$ tends to $\alpha$ as $x$ approaches $b$ through $F$. The verification of this simple statement is left to the reader.

In the following discussion of equicontinuity and normal families of continuous functions we employ the same terminology as in the book of Väisälä [14, $\$ \S 19-20]$. In particular, a sequence $f_{j}: G \rightarrow \overline{\mathbf{R}}^{n}, j=1,2, \ldots$ is said to converge c-uniformly to a mapping $g: G \rightarrow \overline{\mathbf{R}}^{n}$ if it converges uniformly on every compact subset of $G$. A mapping $f: G \rightarrow \overline{\mathbf{R}}^{n}$ of an open half space or an open ball $G$ in $\overline{\mathbf{R}}^{n}$ is said to be normal if, for every sequence $\left(h_{j}\right)$ of conformal self-mappings of $G$, the family $\left(f \circ h_{j}\right)$ is a normal family i.e. contains a $c$-uniformly converging subsequence. The main lemma of this section follows. We introduce the notation $C(e, \varphi)=$ $K(-e, \varphi)+\{e\}$, when $e \in S^{n-1}$ and $\varphi \in(0, \pi / 2)$.

Lemma 3.3. A normal mapping $f: \mathbf{R}_{+}^{n} \rightarrow \overline{\mathbf{R}}^{n}$ has the angular limit $\alpha$ at 0 if and only if the following condition is satisfied:

If $\left(r_{j}\right)$ is a sequence of real numbers in $(0,1)$ tending to zero and if $h_{j}: \mathbf{R}_{+}^{n} \rightarrow \mathbf{R}_{+}^{n}$ is the dilation $h_{j}(x)=x / r_{j}, j=1,2, \ldots$, then every c-uniformly converging subsequence of $\left(f \circ h_{j}^{-1}\right)$ converges to the constant $\alpha$.

Proof. Suppose first that $f$ has the angular limit $\alpha$ at 0 . Since $f$ is normal, there is a subsequence of $\left(f \circ h_{j}^{-1}\right)$, denoted again by $\left(f \circ h_{j}^{-1}\right)$, which converges $c$-uniformly to $g: \mathbf{R}_{+}^{n} \rightarrow \overline{\mathbf{R}}^{n}$. To show that $g \equiv \alpha$ fix $x_{0} \in \mathbf{R}_{+}^{n}$. Let $\varphi \in(0, \pi / 2)$ be such that $x_{0} \in C\left(e_{n}, \varphi\right)$. The sequence $\left(h_{j}^{-1}\left(x_{0}\right)\right)$ tends to the origin through the cone $C\left(e_{n}, \varphi\right)$ and therefore $f \circ h_{j}^{-1}\left(x_{0}\right)$ tends to $\alpha$ as $j \rightarrow \infty$. Hence $g\left(x_{0}\right)=\alpha$ and since $x_{\mathbf{0}} \in \mathbf{R}_{+}^{n}$ was arbitrary, $g$ is the constant $\alpha$.

To prove sufficiency assume that there is $\varphi \in(0, \pi / 2)$ and a sequence $\left(x_{j}\right)$ in $C\left(e_{n}, \varphi\right) \cap B^{n}$ with $x_{j} \rightarrow 0$ and $f\left(x_{j}\right) \rightarrow \beta \neq \alpha$ as $j \rightarrow \infty$. Define $h_{j}: \mathbf{R}_{+}^{n} \rightarrow \mathbf{R}_{+}^{n}$ by $h_{j}(x)=x /\left|x_{j}\right|, j=1,2, \ldots$. Since $f$ is normal, there is a subsequence of $\left(f \circ h_{j}^{-1}\right)$, denoted again by $\left(f \circ h_{j}^{-1}\right)$, which converges $c$-uniformly to a limit mapping $g$ : $\mathbf{R}_{+}^{n} \rightarrow \overline{\mathbf{R}}^{n}$. By hypothesis, $g$ must be the constant $\alpha$. Set $K=\overline{C\left(e_{n}, \varphi\right)} \cap S^{n-1}$. Then 
$h_{j}\left(x_{j}\right) \in K$ for all $j$ and $\left(f \circ h_{j}^{-1}\right)\left(h_{j}\left(x_{j}\right)\right) \rightarrow \beta$ as $j \rightarrow \infty$. This contradicts the convergence of $\left(f \circ h_{j}^{-1}\right)$ to $\alpha$ on $K$. The proof is complete.

The next result follows immediately from the well known equicontinuity properties of quasiconformal mappings and from Ascoli's theorem (cf. [14, 19.2, 20.4]).

Corollary 3.4. A quasiconformal mapping $f: \mathbf{R}_{+}^{n} \rightarrow \overline{\mathbf{R}}^{n}$ is normal and hence has the angular limit $\alpha$ at 0 if and only if the criterion of Lemma 3.3 is satisfied.

\section{Lindelöf-type theorems}

In the case of bounded analytic functions of the unit disc there exist various results guaranteeing the existence of an angular limit at a given boundary point. The fundamental result of this kind, due to Lindelöf, states that the existence of an asymptotic value implies the existence of an angular limit. Subsequently the hypothesis of the existence of an asymptotic value has been weakened by J. L. Doob [2, Thm. 4], T. Hall [5, Thm. II], and by others.

For quasiconformal mappings in $n$-space the only well known result of this sort is the following theorem of Gehring [3, p. 21]. The original proof was given in the case $n=3$ and the general case can be proved in the same way. For alternative proofs we refer the reader to Näkki [12] and to $[15,6.4]$.

Lemma 4.1. Let a quasiconformal mapping $f: B^{n} \rightarrow G^{\prime}$ have an asymptotic value $\alpha$ at $b \in \partial B^{n}$. Then $f$ has the angular limit $\alpha$ at $b$.

In the hypothesis of Lemma 4.1, the assumption of the existence of an asymptotic value will be weakened to the requirement that $f(x)$ tend to a limit $\alpha$ as $x$ approaches $b \in \partial B^{n}$ through a set $E \subset B^{n}$. In order to conclude that $\alpha$ is then the angular limit of $f$ at $b$, it is obviously necessary to require that the set $E$ not be too thin at $b$. Theorem 4.4 shows that such a condition can be expressed in terms of the lower capacity density by requiring cap dens $(E, b)>0$. Then we use this result to deduce Theorem 4.7, the quasiconformal analogue of T. Hall's theorem. First we give some auxiliary results.

Lemma 4.2. Let $D$ be an open half space or an open ball in $\overline{\mathbf{R}}^{n}$ and let $E$ and $F$ be subsets of $\bar{D}$. Then

$$
M(\Delta(E, F ; \bar{D})) \geqq \frac{M\left(\Delta\left(E, F ; \overline{\mathbf{R}}^{n}\right)\right)}{2} .
$$

Proof. In view of the conformal invariance of the modulus (cf. $[14,8.1]$ ) we may assume that $D=\mathbf{R}_{+}^{n}$. Write $\Gamma_{+}=\Delta\left(E, F ; \overline{\mathbf{R}}_{+}^{n}\right)$ and $\Gamma=\Delta\left(E, F ; \overline{\mathbf{R}}^{n}\right)$. Let $\varrho_{+} \in F\left(\Gamma_{+}\right)$and let $\varphi: \overline{\mathbf{R}}^{n} \rightarrow\{0,1\}$ be the characteristic function of $\bar{D}$. Then 
$\varrho_{+}=\varphi \cdot \varrho_{+} \in F\left(\Gamma_{+}\right)$and we define $\varrho: \mathbf{R}^{n} \rightarrow \mathbf{R}^{1} \cup\{\infty\}$ by $\varrho \mid \mathbf{R}_{+}^{n}=\tilde{\varrho}_{+}$and $\varrho\left(\left(x_{1}, \ldots, x_{n-1},-x_{n}\right)\right)=\tilde{\varrho}_{+}\left(\left(x_{1}, \ldots, x_{n-1}, x_{n}\right)\right)$ for $x_{n} \geqq 0$. Then we see that $\varrho \in F(\Gamma)$ and obtain

$$
M(\Gamma) \leqq \int_{\mathbf{R}^{n}} \varrho^{n} d m_{n}=2 \int_{\mathbf{R}^{n}} \tilde{\varrho}_{+}^{n} d m_{n} \leqq 2 \int_{\mathbf{R}^{n}} \varrho_{+}^{n} d m_{n}
$$

Since $\varrho_{+} \in F\left(\Gamma_{+}\right)$was arbitrary, these estimates show that $M(\Gamma) \leqq 2 M\left(\Gamma_{+}\right)$, as desired.

The next lemma yields a particular type of symmetry property for the modulus. For related results we refer the reader to Gehring [4, Lemma 1] and to the references given in [4].

Lemma 4.3. Let $D$ be an open half space or an open ball in $\overline{\mathbf{R}}^{n}$ and let $E$ and $F$ be subsets of $D$. Then

$$
M(\Delta(E, F ; D)) \geqq \frac{M\left(\Delta\left(E, F ; \overline{\mathbf{R}}^{n}\right)\right)}{2} .
$$

Proof. By $[14,8.1]$ we may assume that $D=B^{n}$. Let $E_{j}=E \cap \bar{B}^{n}(1-1 / j)$, $F_{j}=F \cap \bar{B}^{n}(1-1 / j), \Gamma=\Delta\left(E, F ; B^{n}\right), \quad \Gamma_{j}=\Delta\left(E_{j}, F_{j} ; \bar{B}^{n}(1-1 / j)\right), \quad \Delta=\Delta\left(E, F ; \overline{\mathbf{R}}^{n}\right)$, and $\left.\Delta_{j}=\Delta\left(E_{j}, F_{j} ; \overline{\mathbf{R}}^{n}\right)\right)$ for $j=2,3, \ldots$ With this notation we get by Lemma 4.2

$$
M(\Gamma) \geqq M\left(\cup \Gamma_{j}\right) \geqq \lim M\left(\Gamma_{j}\right) \geqq \frac{\lim M\left(\Delta_{j}\right)}{2}=\frac{M(\Delta)}{2} .
$$

In the last step we have used the obvious equality $\Delta=\cup \Delta_{j}$ and a result of Ziemer [19, Lemma 2.3] for the moduli of increasing path families.

Now we shall prove the main result of this section.

Theorem 4.4. Let $f: B^{n} \rightarrow G^{\prime}$, be a quasiconformal mapping and let $f(x)$ tend to a limit $\alpha$ as $x$ approaches $b \in \partial B^{n}$ through a set $E$ in $B^{n}$ with cap dens $(E, b)>0$. Then $f$ has the angular limit $\alpha$ at $b$.

Proof. By performing preliminary Möbius transformations we may, in view of Lemma 1.14 , replace $B^{n}$ and $b$ by $\mathbf{R}_{+}^{n}$ and 0 and make $\alpha$ finite. Let $\left(r_{j}\right)$ be a sequence of real numbers in $(0,1)$ tending to 0 and for $j=1,2, \ldots$ let $h_{j}: \mathbf{R}_{+}^{n} \rightarrow \mathbf{R}_{+}^{n}$ be the dilation $h_{j}(x)=x / r_{j}$. According to Corollary 3.4 it will be enough to show that every $c$-uniformly converging subsequence of $\left(f \circ h_{j}^{-1}\right)$ tends to the constant $\alpha$.

Choose a $c$-uniformly converging subsequence, denoted again by $\left(f \circ h_{j}^{-1}\right)$, which tends to a limit mapping $g: \mathbf{R}_{+}^{n} \rightarrow \overline{\mathbf{R}}^{n}$. From [14, 21.3] it follows that $g$ is a homeomorphism or a constant. Assume that $g$ is not the constant $\alpha$. Then we may fix a continuum $F \subset \mathbf{R}_{+}^{n} \cap \bar{B}^{n}$ and choose $r>0$ such that $g F \subset \overline{\mathbf{R}}^{n} \backslash B^{n}(\alpha, 2 r)$. Since the convergence of $f \circ h_{j}^{-1}$ to $g$ is $c$-uniform, we may choose an integer $j_{0}$ such that $f \circ h_{j}^{-1} F \subset \overline{\mathbf{R}}^{n} \backslash B^{n}(\alpha, r)$ for all $j \geqq j_{0}$. Let $E_{j}=E \cap \bar{B}^{n}\left(r_{j}\right)$ and $\Gamma_{j}=$ $\Delta\left(F, h_{j} E_{j} ; \mathbf{R}_{+}^{n}\right)$ for $j \geqq j_{0}$. Since $h_{j} E_{j}=\bar{B}^{n} \cap h_{j} E$ we see that $M\left(\Delta\left(F, \bar{B}^{n} \cap h_{j} E ; \mathbf{R}_{+}^{n}\right)\right)=$ 
$M\left(\Gamma_{j}\right)$ for $j \geqq j_{\mathfrak{c}}$. Hence there exists by Lemmas 1.12 and 4.3 a constant $\mu>0$ depending only on $F$ and $n$ such that

$$
\liminf _{j \rightarrow \infty} M\left(\Gamma_{j}\right) \geqq \frac{\mu \operatorname{cap} \operatorname{dens}(E, 0)}{2}>0 .
$$

Let $f_{j}=f \circ h_{j}^{-1}$ for $j \geqq j_{0}$. Since $f_{j} F \subset \overline{\mathbf{R}}^{\natural} \backslash B^{n}(\alpha, r)$ for $j \geqq j_{0}$ and $f_{j}\left(h_{j} E_{j}\right) \rightarrow \alpha$ as $j \rightarrow \infty$, it follows from (1.8) that $M\left(f_{j} \Gamma_{j}\right) \rightarrow 0$ as $j \rightarrow \infty$. By the modulus inequality (1.3) we get

$$
M\left(\Gamma_{j}\right) \leqq K\left(f_{j}\right) M\left(f_{j} \Gamma_{j}\right)
$$

For sufficiently large $j$ this inequality contradicts the inequality just obtained for lim inf $M\left(\Gamma_{j}\right)$, since the conformality of the mappings $h_{j}$ implies that $K\left(f_{j}\right)=$ $K(f) \in[1, \infty)$ is independent of $j$. We see that $g$ must be the constant $\alpha$, and the proof is complete.

Remarks 4.5.

(1) Example 6.6 shows that in Theorem 4.4 the condition cap dens $(E, b)>0$ cannot be replaced by cap $\overline{\text { dens }}(E, b)>0$. However, the condition can be weakened, as we shall show in Theorem 5.5.

(2) We shall show in 6.14 that, in the particular case when $E$ is non-tangential in the sense of 6.14, Theorem 4.4 follows from Corollary 6.5.

We shall now give some consequences of Theorem 4.4. For further consequences we refer the reader to Section 6. First we recall that by Remark 3.2 there is no loss of generality in assuming that the set $E$ be open. Observe that the densities in question are defined, since $E$ is open.

Corollary 4.6. Let $f: B^{n} \rightarrow G^{\prime}$ be a quasiconformal mapping and let $f(x)$ tend to a limit $\alpha$ as $x$ approaches $b \in \partial B^{n}$ through an open set $E$ in $B^{n}$. Then $f$ has the angular limit $\alpha$ at $b$ if one of the following conditions is satisfied:

(i) $\operatorname{rad}$ dens $(E, b)>0$,

(ii) For some $k, 1 \leqq k \leqq n$, there is a $k$-dimensional affine subspace $V$ through $b$ and an orthogonal projection $p: \mathbf{R}^{n} \rightarrow V$ such that $\Theta_{*}^{k}(p E, b)>0$.

Proof. The proof follows from Theorem 4.4 and Lemmas 2.2 and 2.4.

The next theorem is a quasiconformal version of T. Hall's theorem [5, Thm. II].

Theorem 4.7. Let $f: \mathbf{R}_{+}^{n} \rightarrow G^{\prime}$ be a quasiconformal mapping and let $f(x)$ tend to a limit $\alpha$ as $x$ approaches $\infty$ through an open set $E \subset \mathbf{R}_{+}^{n}$ with

$$
\liminf _{r \rightarrow \infty} m(A \cap(0, r)) r^{-1}>0
$$

where $A=\left\{r>0: E \cap S^{n-1}(r) \neq \emptyset\right\}$. Then for each $\varphi \in(0, \pi / 2) f(x)$ tends to $\alpha$ as $x$ approaches o through the cone $C\left(e_{n}, \varphi\right)$. 
Proof. Let $h: \overline{\mathbf{R}}^{n} \rightarrow \overline{\mathbf{R}}^{n}$ be the inversion $h(x)=x /|x|^{2}$ for $x \in \mathbf{R}^{n} \backslash\{0\}$ and $h(0)=\infty, h(\infty)=0$. Then $h \mathbf{R}_{+}^{n}=\mathbf{R}_{+}^{n}$. It is left to the reader to show that rad dens $(h E, 0)>0$. The proof now follows from Corollary 4.6 (i).

Remark 4.8. As is seen by Example 2.5 (3) the assumption $\operatorname{rad}$ dens $(E, b)>0$

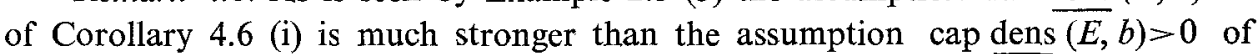
Theorem 4.4 (cf. also Example 6.9 (3)). Hence, in the very special case when $f$ is conformal and $n=2$, one obtains from Theorem 4.4 a stronger result than what T. Hall's theorem [5, Thm. II] gives for $f$ conformal. Even this special case of Theorem 4.4 seems to be new.

\section{Counterparts of a theorem of J. L. Doob}

Given a continuous mapping $f: B^{n} \rightarrow \mathbf{R}^{n}, \alpha \in \overline{\mathbf{R}}^{n}$, and $\varepsilon>0$ we let $E_{\varepsilon}$ denote the set $\left\{x \in B^{n}:|f(x)-\alpha|<\varepsilon\right\}$ for $\alpha \neq \infty$ and the set $\left\{x \in B^{n}:|f(x)|>1 / \varepsilon\right\}$ for $\alpha=\infty$. Clearly, the sets $E_{\varepsilon}$ are open for all $\varepsilon>0$. The cluster set $C(f, b)$ of $f$ at $b \in \partial B^{n}$ is the set of all such values of $\alpha$ for which $b \in \bar{E}_{\varepsilon}$ for all $\varepsilon>0$. From the definition it follows that $\alpha \in C(f, b)$ if and only if there is a sequence $\left(b_{k}\right)$ in $B^{n}$ with $b_{k} \rightarrow b$ and $f\left(b_{k}\right) \rightarrow \alpha$ as $k \rightarrow \infty$.

In the present section we shall improve Theorem 4.4 by giving a rather general sufficient condition for the fact that $\alpha \in C(f, b)$ is the angular limit of a quasiconformal mapping $f: B^{n} \rightarrow G^{\prime} \subset \mathbf{R}^{n}$ at $b \in \partial B^{n}$. In this discussion the sets $E_{\varepsilon}, \varepsilon>0$, will play a role similar to that of the set $E$ in Theorem 4.4 and the condition cap dens $(E, b)>0$ of 4.4 will be replaced by the more general condition that cap $\overline{\text { dens }}\left(E_{\varepsilon}, b\right)$ do not tend too rapidly to 0 as $\varepsilon$ tends to 0 . In [2] J. L. Doob proved results of this kind for bounded analytic functions. Our methods are quite different from those of Doob and are based on the results of Sections $1-3$. Following the ideas of Doob's paper we introduce now some terminology.

Let $f: B^{n} \rightarrow \mathbf{R}^{n}$ be continuous, let $b \in \partial B^{n}, \alpha \in C(f, b)$, and let $\delta_{\varepsilon}=$ cap dens $\left(E_{\varepsilon}, b\right)$. Then $f$ has a capacity cluster value $\alpha$ at $b$ if

$$
\lim _{\varepsilon \rightarrow 0} \varepsilon^{\left(\delta_{\varepsilon}\right)^{d}}=0
$$

for some $d>0$. The mapping $f$ has a metric cluster value $\alpha$ at $b$ if condition (5.1) holds with $\delta_{\varepsilon}=\operatorname{rad}$ dens $\left(E_{\varepsilon}, b\right)$. It follows from Lemma 2.2 that a metric cluster value is a capacity cluster value as well. The least upper bound of numbers $d>0$ for which (5.1) holds is called the order of the cluster value $\alpha$. Observe that if the order is greater than $d^{\prime}$, then condition (5.1) holds with $d$ replaced by $d^{\prime}$. We also define the capacity and metric cluster values of $f$ at $b$ on a straight line $L$ by requir- 
ing that (5.1) holds with $\delta_{\varepsilon}=$ cap dens $\left(E_{\varepsilon} \cap L, b\right)$ and $=\operatorname{rad} \underline{\text { dens }}\left(E_{\varepsilon} \cap L, b\right)$, respectively.

J. L. Doob has proved the following theorem in [2, Thm. 4].

Lemma 5.2. Let a bounded analytic function $f: B^{2} \rightarrow \mathbf{R}^{2}$ have a finite complex value $\alpha$ as a metric cluster value on a straight line at $b \in \partial B^{2}$. If the order of $\alpha$ is greater than 2 then $f$ has an angular limit $\alpha$ at $b$.

We are going to prove a quasiconformal analogue of Lemma 5.2. But first, it is useful to study the connection between capacity cluster values and approximate limits. A mapping $f: B^{n} \rightarrow \mathbf{R}^{n}$ has an approximate limit $\alpha$ at $b \in \partial B^{n}$ if for every $\varepsilon>0$

$$
\lim _{r \rightarrow 0} m_{n}\left(\left(B^{n} \backslash E_{\varepsilon}\right) \cap B^{n}(b, r)\right) r^{-n}=0
$$

where the set $E_{\varepsilon}$ has the meaning explained at the beginning of the section.

Remarks 5.3.

(1) Condition (5.1) restricts the rapidity of approach of $\delta_{\varepsilon}$ to 0 with $\varepsilon$. An important special case is $\delta_{\varepsilon}=$ cap dens $\left(E_{\varepsilon}, b\right) \geqq \delta>0$ for all $\varepsilon>0$, in which case condition (5.1) is satisfied for every $d>0$.

(2) The condition in the above definition of the approximate limit can be expressed in terms of the Lebesgue density by

$$
\Theta^{n}\left(E_{\varepsilon}, b\right)=\Theta^{n}\left(B^{n}, b\right)=\frac{1}{2}
$$

for every $\varepsilon>0$. From Lemma 2.4 it follows that when this equality holds $\operatorname{rad}$ dens $\left(E_{\varepsilon}, b\right) \geqq \delta>0$ for every $\varepsilon>0$ with $\delta$ depending only on $n$. Condition (5.1) is therefore fulfilled in view of (1) and Lemma 2.2. Hence we have seen that an approximate limit is a metric cluster value as well.

The next lemma gives a characterization for the existence of an approximate limit. However, it is not necessary for what follows and is given mainly for completeness. The proof of the lemma is left to the reader.

Lemma 5.4. A mapping $f: B^{n} \rightarrow \mathbf{R}^{n}$ has an approximate limit $\alpha$ at $b \in \partial B^{n}$ if and only if there is an open set $E$ in $B^{n}$ with $\Theta^{n}\left(B^{n} \backslash E, b\right)=0$ and such that $f(x)$ tends to $\alpha$ as $x$ approaches $b$ through the set $E$.

Throughout the remaining part of this section we let the notation $f: B^{n} \rightarrow G^{\prime}$ include the assumption that $G^{\prime} \subset \mathbf{R}^{n}$, whence the sets $E_{\varepsilon} \varepsilon>0$, introduced at the beginning of this section, are defined for such a mapping $f$.

We are now in a position to prove the main result of the paper, which is a farreaching generalization of Gehring's result in Lemma 4.1. 
Theorem 5.5. Let $f: B^{n} \rightarrow G^{\prime}$ be a quasiconformal mapping, let $b \in \partial B^{n}$, and let $E_{\varepsilon}=f^{-1} B^{n}(\varepsilon), \delta_{\varepsilon}=$ cap dens $\left(E_{\varepsilon}, b\right)$ for $\varepsilon>0$. If

$$
\limsup _{\varepsilon \rightarrow 0} \delta_{\varepsilon}\left(\log \frac{1}{\varepsilon}\right)^{n-1}=\infty,
$$

then $f$ has angular limit 0 at $b$.

Proof. By performing a preliminary Möbius transformation we may, in view of Lemma 1.14 , replace $B^{n}$ by $\mathbf{R}_{+}^{n}$ and $b$ by 0 . Let $\left(r_{j}\right)$ be a sequence of real numbers in $(0,1)$ tending to 0 and for $j=1,2, \ldots$ let $h_{j}: \mathbf{R}_{+}^{n} \rightarrow \mathbf{R}_{+}^{n}$ be the dilation $h_{j}(x)=$ $x / r_{j}$. According to Corollary 3.4 it will be enough to show that every $c$-uniformly converging subsequence of $\left(f \circ h_{j}^{-1}\right)$ tends to the constant 0 .

Choose a $c$-uniformly converging subsequence, denoted again by $\left(f \circ h_{j}^{-1}\right)$, which tends to a limit mapping $g: \mathbf{R}_{+}^{n} \rightarrow \overline{\mathbf{R}}^{n}$. By [14, 21.3] $g$ is a homeomorphism or a constant. Assume that $g$ is not the constant 0 . Then we may fix a continuum $F \subset \mathbf{R}_{+}^{n} \cap \bar{B}^{n}$ and choose $r_{0}>0$ such that $g F \subset \overline{\mathbf{R}}^{n} \backslash B^{n}\left(2 r_{0}\right)$. Since the convergence of $f \circ h_{j}^{-1}$ to $g$ is $c$-uniform, there is an integer $j_{0}$ such that $f \circ h_{j}^{-1} F \subset \overline{\mathbf{R}}^{n} \backslash B^{n}\left(r_{0}\right)$ for every $j \geqq j_{0}$. For $r \in\left(0, r_{0}\right)$ write $E_{r}=\left\{x \in \mathbf{R}_{+}^{n}:|f(x)|<r\right\}$ and $\delta_{r}=$ cap dens $\left(E_{r}, 0\right)$. For $j \geqq j_{0}$ and $r \in\left(0, r_{0}\right)$ let $\Gamma_{j}^{r}=\Delta\left(F, h_{j} E_{r} ; \mathbf{R}_{+}^{n}\right)$. Let $\mu>0$ be the constant of Lemma 1.12 depending only on $F$ and $n$. From Lemmas 1.12 and 4.3 it follows that for each $r \in\left(0, r_{0}\right)$ there is an integer $a_{r} \geqq j_{0}$ such that

$$
M\left(\Gamma_{j}^{r}\right) \geqq \frac{\mu \delta_{r}}{3}
$$

for $j \geqq a_{r}$. Write $f_{j}=f \circ h_{j}^{-1}$. Since for $j \geqq j_{0}$ and $r \in\left(0, r_{0}\right) f_{j} F \subset \overline{\mathbf{R}}^{n} \backslash B^{n}\left(r_{0}\right)$ and $f_{j}\left(h_{j} E_{r}\right) \subset B^{n}(r)$ we get by $(1.8)$

$$
M\left(f_{j} \Gamma_{j}^{r}\right) \leqq \omega_{n-1}\left(\log \left(\frac{r_{0}}{r}\right)\right)^{1-n}
$$

for $r \in\left(0, r_{0}\right)$ and $j \geqq a_{r}$. The conformality of the mappings $h_{j}$ implies that $K\left(f_{j}\right)=$ $K(f) \in[1, \infty)$ is independent of $j$. From the modulus inequality (1.3) and the above inequalities we get

$$
0<\left(\frac{3 \omega_{n-1} K(f)}{\mu}\right)^{-1} \leqq \delta_{r}^{-1}\left(\log \left(\frac{r_{0}}{r}\right)\right)^{1-n}
$$

for $r \in\left(0, r_{0}\right)$, where the left hand side is independent of $r$. Letting $r \rightarrow 0$ yields a contradiction.

We shall now give some corollaries to Theorem 5.5. The first corollary is a quasiconformal counterpart of J. L. Doob's theorem (cf. Lemma 5.2).

Corollary 5.6. Let a quasiconformal mapping $f: B^{n} \rightarrow G^{\prime}$ have a capacity cluster value $\alpha$ of order greater than $1 /(n-1)$ at $b \in \partial B^{n}$. Then $f$ has the angular limit $\alpha$ at $b$. 
Proof. Since the order of $\alpha$ is greater than $1 /(n-1)$, condition (5.1) holds with $d=1 /(n-1)$. Hence also the condition of Theorem 5.5 is satisfied.

Corollary 5.7. Let a quasiconformal mapping $f: B^{n} \rightarrow G^{\prime}$ have a metric cluster value $\alpha$ at $b \in \partial B^{n}$. If the order of $\alpha$ is greater than $1 /(n-1)$, then $f$ has the angular limit $\propto$ at $b$.

Proof. From the definitions it follows that condition (5.1) is satisfied with $\delta_{\varepsilon}=\operatorname{rad}$ dens $\left(E_{\varepsilon}, b\right)$ and $d=1 /(n-1)$. Then, by Lemma 2.2 , it is also satisfied with $\delta_{\varepsilon}=$ cap dens $\left(E_{\varepsilon}, b\right)$ and $d=1 /(n-1)$. The proof now follows from Corollary 5.6.

Remarks 5.8.

(1) Theorem 5.5 seems to be new even in the particular case when $n=2$ and $f$ is a conformal mapping, i.e. a univalent aralytic furction (cf. Remark 4.8).

(2) The author does not know, whether the power $n-1$ in Theorem 5.5 is the best possible.

(3) For $n=2$ and $f$ conformal one obtains from Corollary 5.7 a stronger result than from Doob's theorem (Lemma 5.2 above), since the assumptions on $\alpha$ are weaker in Corollary 5.7 (cf. Example 2.5 (3) and Remark 4.8).

Corollary 5.9. A quasiconformal mapping $f: B^{n} \rightarrow G^{\prime}$ has an approximate limit $\alpha$ at $b \in \partial B^{n}$ if and only if it has an angular limit $\alpha$ at $b$.

Proof. The necessity follows from Theorem 5.5 and Remark 5.3 (2) or alternatively from Lemma 5.4 and Corollary 4.6 (ii). The sufficiency holds even for a continuous mapping. In fact, suppose that $f: B^{n} \rightarrow G^{\prime}$ has an angular limit at $b$. If we apply the definition of an angular limit to the cones $K\left(b, \varphi_{k}\right), \varphi_{k}=\pi k /(2(k+1))$ $k=1,2, \ldots$, we see that there is an set $E \subset B^{n}$ with $\Theta^{n}\left(B^{n} \backslash E, b\right)=0$ and $f(x) \rightarrow \alpha$ as $x \rightarrow b$ through $E$. The proof follows from Lemma 5.4.

\section{Sequential limits and the hyperbolic metric}

In this final section we shall complete our study of angular limits. We begin by showing that a quasiconformal mapping of $B^{n}$ tending to a limit through a sequence of points $\left(b_{k}\right)$ in $B^{n}$ with $b_{k} \rightarrow b \in \partial B^{n}$ tends to the same limit through a larger set $E$ consisting of hyperbolic balls centered at $b_{k}, k=1,2, \ldots$ and having a constant hyperbolic radius. This observation has some interesting consequences. Firstly, it enables us to show that in the results of Sections 4 and 5 the lower capacity densities cannot be replaced by the upper ones. Secondly, some corollaries to our earlier results can be obtained. In particular, we extend a result of Bagemihl and Seidel [1] 
on normal meromorphic functions to the case of quasiconformal mappings of $n$-space.

The hyperbolic metric $\varrho$ in $B^{n}$ is defined by the element of length

$$
d \varrho=\frac{|d x|}{1-|x|^{2}} .
$$

If $a$ and $b$ are in $B^{n}$ then $\varrho(a, b)$ denotes the geodesic distance between $a$ and $b$ corresponding to this element of length. We shall exploit the invariance of $\varrho$ with respect to conformal self-mappings of $B^{n}$. If $b \in B^{n}$ and $M>0$ we denote by $D(b, M)$ the hyperbolic ball $\left\{x \in B^{n}: \varrho(b, x)<M\right\}$. The relations between the euclidean and hyperbolic metric will be of importance in the sequel. For our purposes it will be enough to observe that one obtains from (6.1) the following formulas for the quantities $R_{b}$ and $r_{b}$ :

$$
\begin{array}{cc}
R_{b}=\frac{\left(1-|b|^{2}\right) \tanh M}{1-|b| \tanh M} ; & R_{b}=\max \{|z-b|: z \in \partial D(b, M)\}, \\
r_{b}=\frac{\left(1-|b|^{2}\right) \tanh M}{1+|b| \tanh M} ; & r_{b}=\min \{|z-b|: z \in \partial D(b, M)\} .
\end{array}
$$

The second equality gives a sufficient condition for $x \in D(b, M)$ expressed by means of the euclidean metric as follows: $|b-x|<\tanh M(1-|b|)$.

Theorem 6.3. Let $f: B^{n} \rightarrow \mathbf{R}^{n}$ be a discrete open (cf. [7]) normal mapping with the property that if $\left(h_{j}\right)$ is a sequence of conformal self-mappings of $B^{n}$ and $f \circ h_{j}^{-1}$ tends c-uniformly to a limit mapping $g: B^{n} \rightarrow \overline{\mathbf{R}}^{n}$, then the limit mapping $g$ is either discrete open or constant. Let $\left(b_{k}\right)$ be a sequence in $B^{n}$ with $b_{k} \rightarrow b \in \partial B^{n}$ and $f\left(b_{k}\right) \rightarrow \alpha$ when $k \rightarrow \infty$ and let $M \in(0, \infty)$. If $\alpha \in \partial f B^{n}$ then $f(x)$ tends to $\alpha$ as $x$ approaches $b$ through the set $\cup D\left(b_{k}, M\right)$.

Proof. Let $h_{k}: B^{n} \rightarrow B^{n}$ be a conformal self-mapping of $B^{n}$ with $h_{k}\left(b_{k}\right)=0$. By the conformal invariance of $\varrho, h_{k} D\left(b_{k}, M\right)=D(0, M)$ for $k=1,2, \ldots$ Assume that there is a sequence $\left(a_{k}\right)$ in $\cup D\left(b_{k}, M\right)$ converging to $b$ such that $f\left(a_{k}\right) \rightarrow \beta \neq \alpha$. Since $f$ is normal there is a subsequence of $\left(f \circ h_{k}^{-1}\right)$, which we also denote by ( $f \circ h_{k}^{-1}$ ), converging $c$-uniformly to a limit mapping $g: B^{n} \rightarrow \overline{f B}^{n}$. Since $f \circ h_{k}^{-1}(0)=$ $f\left(b_{k}\right) \rightarrow \alpha$ as $k \rightarrow \infty, g(0)=\alpha$. Since $\alpha \in \partial f B^{n}$, it follows from [7, 2.12], the properties of the local topological index, and from the c-uniform convergence, that $g \equiv \alpha$. After relabeling we may assume that $a_{k} \in D\left(b_{k}, M\right), k=1,2, \ldots$ and that $h_{k}\left(a_{k}\right)$ converges to a limit point $u \in \overline{D(0, M)} \subset B^{n}$. Moreover,

$$
\left(f \circ h_{k}^{-1}\right)\left(h_{k}\left(a_{k}\right)\right) \rightarrow \beta \not \neq g(u)=\alpha .
$$

This conclusion contradicts the convergence of $\left(f \circ h_{k}^{-1}\right)$ to $\alpha$ on the set $\overline{D(0, M)}$. The proof is now complete. 
Remark 6.4. Theorem 6.3 generalizes the reasoning in the proof of a result of Bagemihl and Seidel [1, Thm. 1]. The assumption that $\alpha \in \partial f B^{n}$ can be replaced, as in [1, p. 5], by the requirement that $f^{-1}(\alpha)$ be finite. We show that this last assumption cannot be dropped. For this purpose we consider the normal analytic function $f: B^{2} \rightarrow B^{2 \backslash} \backslash\{0\} \quad$ defined by $f(z)=\exp ((z+1) /(z-1))$ when $z \in B^{2}$. Then $C(f, 1)=\bar{B}^{2}$ and $f^{-1}(\alpha)$ is infinite for all $\alpha \in B^{2} \backslash\{0\}$. We fix $\alpha \in B^{2} \backslash\{0\}$ and choose a sequence $\left(b_{k}\right)$ in $B^{n}$ with $b_{k} \rightarrow 1$ and $f\left(b_{k}\right)=\alpha$ for all $k=1,2, \ldots$. It is easy to verify that the conclusion of Theorem 6.3 fails to hold for this mapping $f$ and the sequence $\left(b_{k}\right)$.

Corollary 6.5. Let $f: B^{n} \rightarrow G^{\prime}$ be a quasiconformal mapping, let $\left(b_{k}\right)$ be a sequence in $B^{n}$ such that $b_{k} \rightarrow b \in \partial B^{n}$, and let $f\left(b_{k}\right) \rightarrow \alpha$ when $k \rightarrow \infty$. If $M \in(0, \infty)$, then $f(x)$ tends to $\alpha$ as $x$ approaches $b$ through the set $\cup D\left(b_{k}, M\right)$.

Proof. Since $f$ is injective $C(f, b) \subset \partial f B^{n}$ and hence the condition $\alpha \in \partial f B^{n}$ in Theorem 6.3 is satisfied. The proof now follows from Theorem 6.3 , since a quasiconformal mapping of $B^{n}$ satisfies by $[14,21.3]$ also the other conditions in 6.3 .

We are now in a position to show by an example that the lower radial and capacity densities in Corollary 4.6 (i) and Theorem 4.4 respectively, cannot be replaced by the corresponding upper densities. Since these results are particular cases of Theorem 5.5, the same statement is true of Theorem 5.5.

Example 6.6. Let $f: B^{2} \rightarrow G^{\prime}$ be a conformal mapping having no angular limit at $e_{1} \in \partial B^{2}$. Fix $M \in(0, \infty)$ and choose $\alpha \in C_{\text {rad }}\left(f, e_{1}\right)$, where $C_{\text {rad }}\left(f, e_{1}\right)$ is the cluster set of $f$ on the radius $\left(0, e_{1}\right)$. Then there is a sequence $\left(b_{k}\right)$ on $\left(0, e_{1}\right)$ with $b_{k} \rightarrow e_{1}$ and $f\left(b_{k}\right) \rightarrow \alpha$ as $k \rightarrow \infty$. By Corollary $6.5 f(x)$ tends to $\alpha$ as $x$ approaches $e_{1}$ through the set $E=\cup D\left(b_{k}, M\right)$. Let

$$
r_{k}=\min \left\{\left|z-b_{k}\right|: z \in \partial D\left(b_{k}, M\right)\right\}, \quad k=1,2, \ldots
$$

By (6.2) we get for all $k$

$$
\frac{r_{k}}{1-\left|b_{k}\right|}=\frac{\left(1+\left|b_{k}\right|\right) \tanh M}{1+\left|b_{k}\right| \tanh M} \geqq \tanh M>0 .
$$

Thus $\operatorname{rad} \overline{\text { dens }}\left(E, e_{1}\right)>0$ (cf. Example $2.5(2)$ ). Since $f(x)$ tends to the limit $\alpha$ as $x$ approaches $e_{1}$ through the set $E$ with $\operatorname{rad} \overline{\operatorname{dens}}\left(E, e_{1}\right)>0$, but $f$ has no angular limit at $e_{1}$, we see that the condition $\operatorname{rad} \operatorname{dens}(E, b)>0$ in Corollary 4.6 (i) cannot be replaced by $\operatorname{rad} \overline{\operatorname{dens}}(E, b)>0$. A corresponding statement concerning the lower capacity density clearly holds in regard to Theorem 4.4.

By combining Corollary 6.5 with Theorem 4.4 and with Gehring's result in Lemma 4.1 above, we obtain some new results. The first result is a quasiconformal 
version of a theorem of Bagemihl and Seidel [1, Thm. 1] concerning normal meromorphic functions.

Corollary 6.7. Let $f: B^{n} \rightarrow G^{\prime}$ be a quasiconformal mapping, let $\left(b_{k}\right)$ be a sequence in $B^{n}$ converging to $b \in \partial B^{n}$ and satisfying the condition

$$
\varrho\left(b_{k}, b_{k+1}\right)<M, \quad k=1,2, \ldots
$$

for some $M \in(0, \infty)$, and let $f\left(b_{k}\right) \rightarrow \alpha$ as $k \rightarrow \infty$. Then $f$ has the angular limit $\alpha$ at $b$.

V. P. Mićić [10] proved a result related to Corollary 6.7 concerning quasiconformal mappings of $\mathbf{R}_{+}^{3}$. However, he considered only the case where the sequence $\left(b_{k}\right)$ lies in a cone with the vertex at a boundary point, and one can show by modifying the calculations of 6.12 to the case of $\mathbf{R}_{+}^{3}$ that his result follows from Corollary 6.7. Mićić proved his theorem by using Gehring's distortion result given in [3].

Corollary 6.8. Let $f: B^{n} \rightarrow G^{\prime}$ be a quasiconformal mapping and let $f(x)$ tend to a limit $\alpha$ as $x$ approaches $b \in \partial B^{n}$ through a set $E \subset B^{n}$ with $b \in \bar{E}$. If there is $M \in(0, \infty)$ such that cap dens $\left(E_{M}, b\right)>0$, where $E_{M}$ denotes the open set $\left\{x \in B^{n}: \varrho(E, x)<M\right\}$, then $f$ has the ungular limit $\alpha$ at $b$.

The sequence $\left(b_{k}\right)$ of Corollary 6.7 has a role similar to that of the set $E$ in Section 4 (cf. Theorem 4.4 and Corollary 4.6) and it may therefore be of some interest to compare the condition of Corollary 6.7 with those in Section 4 . Here we shall show that the condition rad dens $(E, b)>0$ of Corollary 4.6 (i) permits the set $E$ to be very sparse in the sense of the hyperbolic metric.

\section{Examples 6.9.}

(1) Construction of a set $E \subset B^{n}$ with $\operatorname{rad} \underline{\text { dens }}\left(E, e_{1}\right)>0$ such that

$\lim \sup \varrho\left(b_{k}, b_{k+1}\right)=\infty$ for every sequence $\left(b_{k}\right)$ in $E$ tending to $e_{1}$.

First observe that for $b \in B^{n}$ and $M>0$ we obtain from (6.2)

$$
\frac{R_{b}}{1-|b|} \leqq \frac{2 \tanh M}{1-\tanh M}=e^{2 M}-1
$$

Let $t_{2 k}=1-e^{-7 k}$ and write for $k=1,2, \ldots$

$$
E_{2 k}=\left\{x:|x|=t_{2 k}, 2^{-2 k} \leqq\left|x-e_{1}\right| \leqq 2^{-2 k+1}\right\} \text {. }
$$

Since by (6.10) $R_{b} \leqq e^{2 M}(1-|b|)$, it follows that the sets $\left\{z \in B^{n}: \varrho\left(E_{2 k}, z\right)<2 k\right\}$ are disjoint, and thus $\varrho\left(E_{2 k}, E_{2 k+2}\right)>4 k, k=1,2, \ldots$ The construction shows that the set $E=\cup E_{2 k}$ has the desired properties.

(2) In order to illustrate the difference between Corollaries 6.7 and 6.8 we shall construct with the aid of Example (1) a set $F=\left\{b_{k}: k=1,2, \ldots\right\}$ in $B^{n}$ with 
cap dens $\left(F_{M}, e_{1}\right)>0, F_{M}=\cup D\left(b_{k}, M\right)$, for some $M>0$ and with

$$
\lim \sup \varrho\left(b_{k}, b_{k+1}\right)=\infty \text {. }
$$

To this end, fix $M \in(0, \infty)$. For each $k=1,2, \ldots$ choose a finite set $A_{2 k}=$ $\left\{x_{1}^{2 k}, \ldots, x_{p_{k}}^{2 k}\right\} \subset E_{2 k}$ such that

$$
E_{2 k} \subset \bigcup_{j=1}^{P_{k}} D\left(x_{j}^{2 k}, M\right)
$$

Let $\left(b_{k}\right)$ be a sequence such that $\left\{b_{k}: k=1,2, \ldots\right\}=\cup A_{2 k}=F$ and $b_{k} \rightarrow e_{1}$ as $k \rightarrow \infty$. Write $F_{M}=\cup D\left(b_{k}, M\right)$. By (6.11) and by Example (1) $F$ satisfies the condition cap dens $\left(F_{M}, e_{1}\right)>0$ and by Example (1) lim sup $\varrho\left(b_{k}, b_{k+1}\right)=\infty$. Thus the set $F$ has the desired properties.

(3) When we compare Theorem 4.4 and Corollary 6.8 it may be of some interest to know in which way the size of a set $E_{M}=\left\{x \in B^{n}: \varrho(E, x)<M\right\}, M>0$, is related to the size of a set $E \subset B^{n}$ if cap dens $(E, b)>0$ for some $b \in \partial B^{n}$. We shall now construct a set $E \subset B^{n}$ with cap dens $\left(E, e_{1}\right)>0$ and $\operatorname{rad} \overline{\operatorname{dens}}\left(E_{M}, e_{1}\right)=0$ for all $M>0$, provided that the dimension $n \geqq 3$.

Fix $n \geqq 3$. Let $t_{k}=1-2^{-k} / k, k=1,2, \ldots$ and $E=\bigcup_{k=1}^{\infty}\left(S^{n-1}\left(e_{1}, 2^{-k}\right) \cap\right.$ $\left.S^{n-1}\left(t_{k}\right)\right)$. Since $n \geqq 3$, it follows from Lemma 1.10 that cap dens $\left(E, e_{1}\right)=0$. To show that $\operatorname{rad} \overline{\operatorname{dens}}\left(E_{M}, e_{1}\right)=0$ for all $M \in(0, \infty)$, fix $M \in(0, \infty)$. Choose an integer $k_{0}$ so that $2 e^{2 M} / k_{0} \leqq 1 / 4$. Let $A=\left\{r>0: S^{n-1}\left(e_{1}, r\right) \cap E_{M} \neq \emptyset\right\}$. Using the inequality $d(D(b, M)) \leqq 2 e^{2 M}(1-|b|)$ (cf. (6.10)) we can estimate the lengths of the components of $A \cap\left(0,2^{-k_{0}}\right)$. Fix $r \in\left(0,2^{-k_{0}}\right)$ and choose $k_{r} \geqq k_{0}$ such that $r \in\left[2^{-k_{r}-1}, 2^{-k_{r}}\right)$. Then

which tends to zero as $r \rightarrow 0$.

$$
m(A \cap(0, r)) r^{-1} \leqq\left(\sum_{k \geqq k_{r}} \frac{2 e^{2 M} 2^{-k}}{k}\right) 2^{k_{r}+1} \leqq \frac{8 e^{M}}{k_{r}}
$$

In the remaining part of the paper we shall show that an essential feature of Example 6.9 (1) is the tangential approach of the set $E$ to $\partial B^{n}$. For this purpose, a finite upper bound for the hyperbolic diameter of a set is needed.

6.12. An upper bound for the hyperbolic diameter. Fix $\varphi \in(0, \pi / 2), u \in(0,1)$, $b \in \partial B^{n}$, and $t \in(0, \cos \varphi)$. We shall consider the set

$$
A(u, \varphi, t)=K(b, \varphi) \cap\left(B^{n}(b, t) \backslash \bar{B}^{n}(b, u t)\right)
$$

and shall give an upper bound for the hyperbolic diameter of $A(u, \varphi, t)$ independent of $t$ and depending only on $u$ and $\varphi$. From (6.1) it follows that

$$
\varrho(x, y) \leqq \frac{|x-y|}{\min \left\{1-|x|^{2}, 1-|y|^{2}\right\}}
$$


for $x, y$ in $B^{n}$. Since $t \in(0, \cos \varphi)$ we observe that

$$
d\left(\bar{A}, \partial B^{n}\right)=d\left(\bar{A} \cap S^{n-1}(b, u t), \partial B^{n}\right)=1-\sqrt{\sin ^{2} \varphi+(\cos \varphi-u t)^{2}}
$$

where $\bar{A}=\overline{A(u, \varphi, t)}$. Hence $1-|x| \geqq d\left(\bar{A}, \partial B^{n}\right)$ for all $x \in \bar{A}$. Therefore

$$
1-|x|^{2}=(1+|x|)(1-|x|) \geqq u t(2 \cos \varphi-u t) \geqq u t \cos \varphi .
$$

Because $d(\bar{A}) \leqq 2 t$ we obtain in view of (6.13) the upper bound $2 /(u \cos \varphi)$ for the hyperbolic diameter of $A(u, \varphi, t)$.

6.14. Non-tangential sets. Recall that we constructed in Example 6.9 (1) a set $E \subset B^{n}$ with $\operatorname{rad} \underline{\text { dens }}\left(E, e_{1}\right)>0$ and with the property that lim sup $\varrho\left(b_{k}, b_{k+1}\right)=\infty$ for every sequence $\left(b_{k}\right)$ in $E$ tending to $b_{1}$. The above upper bound for the hyperbolic diameter will now be applied to prove that this is possible only for sets approaching $\partial B^{n}$ tangentially. It should be observed that this reasoning shows, in particular, that for non-tangential sets $E$ Theorem 4.4 follows from Corollary 6.5 .

For this purpose let $b \in \partial B^{n}$ and let $E \subset B^{n}$ be a set which is non-tangential at $b$, i.e. $E \subset K(b, \varphi)$ for some $\varphi \in(0, \pi / 2)$ and let cap dens $(E, b)=\delta>0$. This last assumption together with (1.8) implies the existence of a sequence $\left(b_{k}\right)$ in $E$ such that $b_{k} \rightarrow b$ and such that for some number $u \in(0,1)$ depending only on $\delta$ and $n$

$$
b_{k+1} \in A\left(u, \varphi,\left|b_{k}-b\right|\right), \quad k=1,2, \ldots .
$$

Hence 6.12 implies that $\lim \sup \varrho\left(b_{k}, b_{k+1}\right)<M(u, \varphi)<\infty$, proving thus the asserted fact.

We end the paper with a quasiconformal version of a constancy criterion, which, for normal meromorphic functions, is due to Bagemihl and Seidel [1].

Theorem 6.15. Let $f: B^{n} \rightarrow G^{\prime}$ be a quasiconformal mapping, let $\left(b_{k}\right)$ be a sequence in $B^{n}$ with $\left|b_{k}\right| \rightarrow 1$ when $k \rightarrow \infty$, having at least two distinct limit points on $\partial B^{n}$, and such that

$$
\varrho\left(b_{k}, b_{k+1}\right)<M, \quad k=1,2, \ldots
$$

for some $M \in(0, \infty)$. If $f\left(b_{k}\right) \rightarrow \alpha$ as $k \rightarrow \infty$, then $f$ reduces to the constant $\alpha$.

Proof. Suppose, on the contrary, that $f$ is not the constant $\alpha$. Then we may fix a continuum $F \subset B^{n}$ with $\alpha \notin f F$. Choose two distinct limit points $a, b \in \partial B^{n}$ of the sequence $\left(b_{k}\right)$, and write $3 r=|a, b|>0$. Pick out two subsequences $\left(b_{i_{p}}\right)$ and $\left(b_{j_{p}}\right)$ of $\left(b_{k}\right)$ such that $i_{p}<j_{p}<i_{p+1}$ and $\left|b_{i_{p}}-a\right|<r / p,\left|b_{j_{p}}-b\right|<r / p$, for $p=1,2, \ldots$ Let $C_{p} \subset B^{n}$ be a continuum joining $b_{i_{p}}$ and $b_{j_{p}}$ consisting of the geodesic segments (with respect to the hyperbolic metric) from $b_{k}$ to $b_{k+1}$ for each $k, i_{p} \leqq k \leqq j_{p}-1$. Since $d\left(C_{p}\right) \geqq r$ for every $p$, there is by Lemmas $1.10,1.11$, and 4.3 a $\delta>0$ such that $M\left(\Gamma_{p}\right) \geqq \delta$ for every $p$, where $\Gamma_{p}=\Delta\left(F, C_{p} ; B^{n}\right)$. It follows 
from (6.16) and Corollary 6.5 that $f C_{p} \rightarrow \alpha$ as $p \rightarrow \infty$. Hence (1.8) implies that $M\left(f \Gamma_{p}\right) \rightarrow 0$, since $\alpha \notin f F$. By (1.3) we obtain

$$
\delta \leqq M\left(\Gamma_{p}\right) \leqq K(f) M\left(f \Gamma_{p}\right)
$$

for all $p$. Letting $p \rightarrow \infty$ yields a contradiction. The proof is complete.

\section{Acknowledgements}

This research was completed during the academic year 1977-78, when the author was visiting the Mittag-Leffler Institute. The author is grateful to the Royal Swedish Academy of Sciences for its financial support.

\section{References}

1. Bageminl, F. and Seidel, W., Sequential and continuous limits of meromorphic functions, Ann. Acad. Sci. Fenn. Ser. A I 280 (1960), 1-17.

2. Dooв, J. L., The boundary values of analytic functions, Trans. Amer. Math. Soc. 34 (1932), $153-170$.

3. GeHring, F. W., The Carathéodory convergence theorem for quasiconformal mappings in space, Ann. Acad. Sci. Fenn. Ser. A I 336/11 (1963), 1-21.

4. Gehring, F. W., A remark on domains quasiconformally equivalent to a ball, Ann. Acad. Sci. Fenn. Ser. A I 2 (1976), 147-155.

5. Hall, T., Sur la mesure harmonique de certains ensembles, Ark. Mat. Astr. Fys. 25A, No. 28 (1937), 1-8.

6. Lehto, O. and VIRTANEN, K. I., Boundary behaviour and normal meromorphic functions, Acta Math. 97 (1957), 47-65.

7. Martio, O., R.ICKMan, S. and VÄIsÄLÄ, J., Definitions for quasiregular mappings, Ann. Acad. Sci. Fenn. Ser. A I 448 (1969), 1-40.

8. MARTIO, O., RICKMAN, S. and VÄISÄLÄ, J., Distortion and singularities of quasiregular mappings, Ann. Acad. Sci. Fenn. Ser. A I 465 (1970), 1-13.

9. Martio, O. and Sarvas, J., Density conditions in the n-capacity, Indiana Univ. Math. J. 26 (1977), 761-776.

10. MiĆć́, V. P., A theorem of Lindelöf type for quasiconformal mappings in space, Mat. Vesnik 9 (24) (1972), 3-8.

11. Näkкr, R., Extension of Loewner's capacity theorem, Trans. Amer. Math. Soc. 180 (1973), 229-236.

12. NäKkı, R., Prime ends and quasiconformal mappings, J. Analyse Math. 35 (1979), 13-40.

13. Sarvas, J., Symmetrization of condensers in $n$-space, Ann. Acad. Sci. Fenn. Ser. A I 522 (1972), $1-44$.

14. VÄISÄLÄ, J., Lectures on n-dimensional quasiconformal mappings, Lecture Notes in Mathematics 229, Springer-Verlag, Berlin-Heidelberg-New York, 1971.

15. VuORINen, M., Exceptional sets and boundary behavior of quasiregular mappings in $n$-space, Ann. Acad. Sci. Fenn. Ser. A I Math. Dissertationes 11 (1976), 1-44. 
180 M. Vuorinen: On the existence of angular limits of $n$-dimensional quasiconformal mappings

16. Vuorinen, M., On angular limits of closed quasiregular mappings. Proceedings of the First Finnish-Polish Summer School in Complex Analysis, Part II, (Held at Podlesice, Poland, June 16-22, 1977), Edited by J. Lawrynowicz and O. Lehto, University of Lódź, Łódź 1978, 69-74.

17. Vuorinen, M., Capacity densities and angular limits of quasiregular mappings, Trans. Amer. Math. Soc. (to appear).

18. Wallin, H., Metrical characterization of conformal capacity zero, J. Math. Anal. Appl. 58 (1977), 298-311.

19. ZIEMER, W. P., Extremal length and p-capacity, Michigan Math. J. 16 (1969), 43-51.

Received 18 April 1979

Matti Vuorinen Universitets gatan 37 A 8 SF-20100 Åbo 10

Finland 